\title{
Depression among health workers caring for patients with COVID-19 in Egypt
}

\author{
Hayam Mohamed Elgohary ${ }^{1}$, Mohammad Gamal Sehlo ${ }^{1 *} \mathbb{D}$, Medhat Mohamed Bassiony $^{1}$, \\ Usama Mahmoud Youssef ', Dina Sameh Elrafey ${ }^{2}$ and Shimaa Ibrahim Amin ${ }^{1}$
}

\begin{abstract}
Background: Health care workers caring for patients with COVID-19 pandemic are prone to extraordinary stressors and psychological problems. The aim of this study was to estimate the prevalence and risk factors of major depressive disorder among health care providers who are caring for patients with COVID-19.

Methods: Two hundred-seventy of health care workers were screened for depressive symptoms by DASS-21 Questionnaire. Only 152 of the participants accepted to be interviewed using SCID-I for diagnosis of major depressive disorder.

Results: According to DASS-21, 28.1\% of HCWs had mild-to-moderate depressive symptoms, and $64.8 \%$ with severe symptoms. Of 152 who were interviewed using SCID-I, 74.3\% were diagnosed with major depression disorder.

Young age, decreased sleep hours, female sex, past history of a psychiatric disease, fear of COVID-19 infection for themselves or their relatives, and fear of death with COVID-19 for themselves or their relatives were significant predictors for major depressive disorder and its severity.
\end{abstract}

Conclusion: Major depressive disorder is common among HCWs during COVID-19 pandemic. Screening for depression, particularly for young females, and early treatment are recommended.

Keywords: Major depression disorder, Healthcare workers, COVID-19 pandemic, Egypt

\section{Background}

Lower respiratory tract infections are the communicable diseases with the high mortality rate around the world [1]. In December 2019, a highly infectious acute respiratory syndrome caused by a novel coronavirus (SARSCoV-2) emerged in Wuhan, China. On March 11th 2020, the World Health Organization (WHO) declared COVID-19 a pandemic [2].

According to previous studies from SARS or Ebola epidemics, the onset of a sudden and immediately lifethreatening illness may lead to extraordinary stressors on health care workers (HCWs) [3]. Increased workload,

\footnotetext{
*Correspondence: sehlo68@gmail.com

${ }^{1}$ Department of Psychiatry, Faculty of Medicine, Zagazig University, PO

Box 44519, Zagazig, Egypt

Full list of author information is available at the end of the article
}

physical exhaustion, insufficient personal equipment, fear of infection, and the need to make ethically difficult decisions may have dramatic effects on their physical and mental well-being. Isolation, loss of social support and fear of transmission of infection to relatives and friends could make HCWs liable to mental health problems, such as fear, anxiety, insomnia and depression $[4,5]$. Now in COVID-19 era, health care workers are first-line fighters treating patients with COVID-19 and they are exposed to long and distressing work shifts to meet health requirements which may exceed their individual coping skills [6].

Previous studies have reported the prevalence and factors associated with psychological outcomes in HCWs during past infectious disease outbreaks [7-9]. However, the impact of the current COVID-19 pandemic on the 
psychological well-being of medical staff is yet needed to be studied.

There are only few studies that evaluated depression and its risk factors among HCWs worldwide [10], but they used self-rating scales for assessment of depressive symptoms. The aim of the present research is to estimate the prevalence and potential risk factors contributing to major depressive disorder, which diagnosed by structural interview using SCID-I, among Egyptian health care workers who are caring for COVID-19 patients.

\section{Methods}

The data were collected during the period of March-September, 2020. The participants were the HCWs (doctors, nurses, pharmacists, technicians and paramedical workers) who were working in the isolation units for COVID19 patients, at a university hospital in Egypt. Participants were selected using a convenience sampling technique. Inclusion criteria: age 18-60 years, both sexes and who accepted to participate. The sample size was calculated according the Epi Info 6.0, at 80\% power of the study, 95\% confidence level [11], with minimum sample size 260 . We recruited 300 participants to overcome dropout.

The first step: An electronic Google form survey was sent to the participants via their E-mails $(N=300)$. It was designed by the authors to collect: 1-socio-demographic data (age, gender, marital status, residence, level of education, occupation and telephone number) and other clinical data; 2-data related to Depression, Anxiety and Stress Scale (DASS-21) for assessment of depressive symptoms. Prior to conducting the survey, the purpose of the study was explained to participants. Only 270 complete the survey and 30 gave incomplete response, so they were excluded.

The second step: All participants who complete the depression subscale of DASS-21 $(N=270)$ were invited for further evaluation by applying Structured Clinical Interview for DSM-5 disorders (SCID interview) for diagnosis of Major depressive disorder (MDD). The interviews were done in isolation units considering infection control by using the personal protective equipment PPE. Only 152 completed the SCID-I evaluation interview.

\section{Measures}

A. The Depressive subscale of Depression anxiety stress scale (DASS-21): The Depression Anxiety Stress Scale (DASS-21) is a self-report tool containing 21 items that assess depression, anxiety, and stress. There are subscales with 7 items each (depression, anxiety and stress). Each item is scored on a 4-point Likert scale $(0=$ did not apply to me at all and $3=$ applied to me very much, or most of the time), with a higher score indicating more severe levels of distress. The summation of each scale, then multiplied by two to convert to full scale scores. Each score ranged from 0 to 42 . Participants with cutoff scores of $\geq 10$ for the depression dimension $(\geq 10$ as "mild depression", $\geq 14$ as "moderate", $\geq 21$ as "severe", and $\geq 28$ as "extremely severe"), $\geq 8$ in anxiety ( $\geq 8$ as "mild anxiety", $\geq 10$ as "moderate", $\geq 15$ as "severe", and $\geq 20$ as "extremely severe"), and $\geq 15$ in stress ( $\geq 15$ as "mild anxiety", $\geq 19$ as "moderate", $\geq 26$ as "severe", and $\geq 34$ as "extremely severe") were considered to have these disorders. The scale has been shown to have good test-retest reliability, internal consistency and convergent validity [12].

B. Structured Clinical Interview for DSM-5 disorders, clinician version (SCID-5-CV). For diagnosis of Major depressive disorder [13]

Data analysis was performed using the statistical package for social sciences software (SPSS version 20). The qualitative data were presented in the form of number and percentage. The quantitative data were presented in the form of mean and standard deviation. Groups were compared using independent sample $t$ test for quantitative parameters. For qualitative variables, chi-square was used as a test of significance of differences among groups. Linear and logistic regression was used in the analysis of the predictors for depression. A $P$-value $<0.05$ was considered to indicate statistical significance.

\section{Results}

This study included 270 health care workers (HCWs). The studied group age ranged from 18 to 52 years with mean $34.98 \pm 6.27$ years. $57 \%$ of HCWs were female. More than $72 \%$ of them had post-graduate education. Most frequent occupations among the participants were physicians and nurses $(70.7 \%$ and $16.3 \%$, respectively). The most common specialties among physicians were clinical pathology, anesthesia, ICU and pulmonary $(10.4 \%, 10 \%$ and $7 \%$, respectively). Most of the $\mathrm{HCWs}$ were married (79.6\%), from urban area (77.4\%) and non-smokers (84.4\%). Only 9.6\% of the studied HCWs had past history of psychiatric disorders and $12.6 \%$ had family history of psychiatric disorders.

Among HCWs, 2.2\% reported suicidal thoughts. The working hours ranged from 4 to 14 with mean $9.31 \pm 3.1$ while sleeping hours ranged from 3 to 10 with mean $6.57 \pm 1.27$. DASS- 21 was answered by 270 of the participants (response rate: $90 \%$ ). HCWs without depressive symptoms were $7 \%(N=19)$, mild $13.3(N=36)$, moderate $14.8 \%(N=40)$, severe $18.5 \%(N=50)$ and extremely severe depressed HCWs represented $46.3 \%(N=125)$ as shown in Fig. 1. 


\section{Depression}

$\mathrm{N}=19$

(7.1\%)

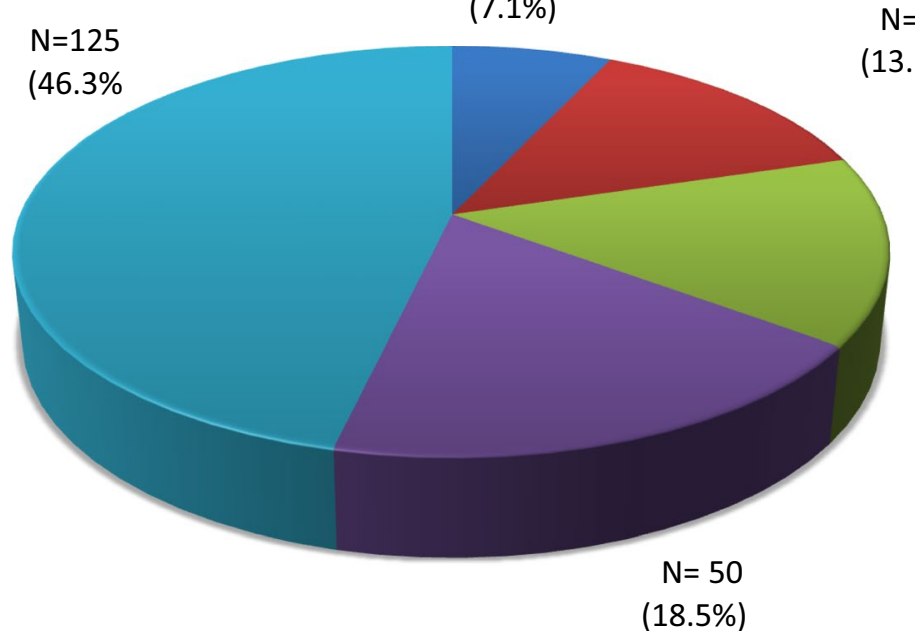

$\mathrm{N}=40$

$(14.8 \%)$

$(18.5 \%)$

Fig. 1 Frequency of depressive symptoms according to DASS-21 questionnaire among the HCWs

Table 1 shows the comparison between HCWs with severe-to-extremely severe depression and those with mild-to-moderate depression. There was a statistical significant decrease in mean age and sleep hours among HCWs with severe and extremely severe depression. In addition, there was a statistical significant increase in frequency of severe and extremely severe depression among females, HCWs who had past history of psychiatric disorder, fear of working during COVID-19 pandemic, fear of being infected with COVID-19, fear of infection of a family member with COVID-19, fear of death with COVID-19 and fear of a family member death with COVID-19. The same factors remain significant after linear regression analysis for predictors of depression severity according to DASS-21 score as shown in Table 2.

SCID-I evaluation was conducted on $152 \mathrm{HCWs}$ who reported depression by DASS-21 scale and the evaluation revealed that 113 of them (74.3\%) diagnosed with major depression (Fig. 2).

Depressed HCWs had statistically decreased mean age and decreased sleep hours than non-depressed. Moreover, frequency of depression was statistically increased among females, those who had a past history of psychiatric disorders, presence of fear of being infected with COVID-19, fear of infection of a family member with COVID-19, fear of death with COVID-19 and fear of a family member death with COVID-19 as shown in Table 3 . The same factors remain significant after applying binary logistic regression analysis as shown in Table 4 .

\section{Discussion}

To our knowledge, this is the first research to use the structured clinical interview for DSM (SCID-I) to detect major depression disorder (MDD) among health care workers (HCWs) who were treating patients with COVID-19 in Egypt.

The current study was a cross-sectional survey that enrolled $270 \mathrm{HCWs}$ and revealed a high prevalence of depressive disorder. Overall respondents, 251(93\%) had depressive symptoms based on DASS-21. Twentyeight percent of HCWs had mild-to-moderate depressive symptoms, and $65 \%$ with severe or extremely severe depressive symptoms. By applying SCID-I, 74.3\% of $\mathrm{HCW}$ were diagnosed with MDD. In a previous study during the acute SARS outbreak, $89 \%$ of HCWs who were in high-risk situations reported psychological symptoms [14]. An Egyptian study similarly reported a high prevalence of severe-to-extremely severe depressive symptoms among Egyptian physicians during the COVID-19 pandemic, the majority (63\%) suffered from severe or extremely severe depressive symptoms [15]. Also, the prevalence of depressive symptoms among health care providers was (78.1\%) in Jordan [16].

Two Egyptian studies and another Saudi one reported that severe depression represents $20.5 \%, 14 \%, 5.8 \%$, consequently these results are considered low in comparison to the current one. This can be explained by the using of different measures noting the in this study the authors used the confirmatory diagnostic clinical interview according to the DSM [17-19]. 
Table 1 Comparison between those who are normal, mild and moderate depression and those with severe and extremely severe depression according to DASS-21 depression score

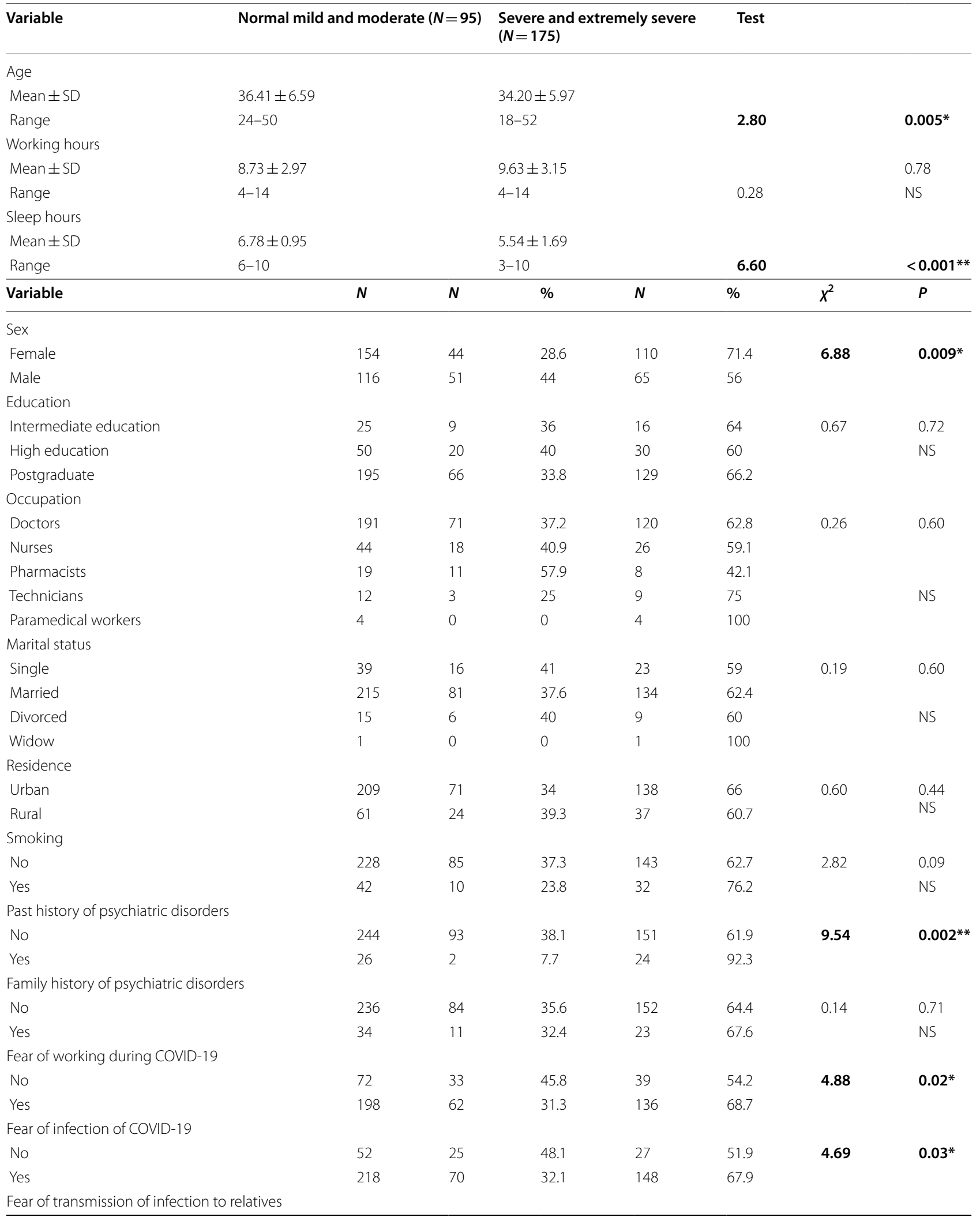


Table 1 (continued)

\begin{tabular}{|c|c|c|c|c|c|c|c|}
\hline Variable & $N$ & $N$ & $\%$ & $N$ & $\%$ & $x^{2}$ & $P$ \\
\hline No & 10 & 6 & 60 & 4 & 40 & 8.05 & 0.004 \\
\hline Yes & 260 & 56 & 21.6 & 204 & 78.4 & & \\
\hline \multicolumn{8}{|c|}{ Had infected with COVID-19 } \\
\hline No & 253 & 93 & 36.7 & 160 & 63.3 & 0.13 & 0.71 \\
\hline Yes & 17 & 7 & 41.2 & 10 & 58.8 & & NS \\
\hline \multicolumn{8}{|c|}{ Had family member infected with COVID-19 } \\
\hline No & 216 & 100 & 46.2 & 116 & 53.8 & 0.13 & 0.71 \\
\hline Yes & 54 & 24 & 18.5 & 30 & 81.5 & & NS \\
\hline \multicolumn{8}{|c|}{ Fear of death with COVID-19 } \\
\hline No & 96 & 42 & 43.8 & 54 & 56.2 & 4.79 & $0.03^{*}$ \\
\hline Yes & 174 & 53 & 30.5 & 121 & 69.5 & & \\
\hline \multicolumn{8}{|c|}{ Fear of family member death with COVID-19 } \\
\hline No & 19 & 12 & 63.2 & 7 & 36.8 & 7.70 & 0.005 \\
\hline Yes & 251 & 80 & 35.1 & 171 & 64.9 & & \\
\hline \multicolumn{8}{|c|}{ Suicidal thoughts } \\
\hline No & 264 & 98 & 37.1 & 166 & 62.9 & 3.50 & 0.06 \\
\hline Yes & 6 & 0 & 0 & 6 & 100 & & NS \\
\hline
\end{tabular}

Bold values highlight the significant results

$S D$ standard deviation, Test independent $t$ test, $X^{2}$ chi-square test

NS non-significant $(P>0.05)$

*Significant $(P<0.05)$; **Highly significant $(P<0.001)$

Table 2 Linear regression analysis for predictors for severity of depression according to DASS-21 depression score among the studied group

\begin{tabular}{|c|c|c|c|c|c|c|c|}
\hline & \multicolumn{2}{|c|}{$\begin{array}{l}\text { Unstandardized } \\
\text { coefficients }\end{array}$} & \multirow{2}{*}{$\begin{array}{l}\text { Standardized } \\
\text { coefficients } \\
\text { Beta }\end{array}$} & \multirow[t]{2}{*}{$t$} & \multirow[t]{2}{*}{$P$} & \multirow[t]{2}{*}{$95.0 \% \mathrm{Cl}$} & \\
\hline & $B$ & SE & & & & & \\
\hline Age & -0.38 & 0.09 & -0.24 & -4.23 & $<0.001^{* *}$ & -0.56 & -0.20 \\
\hline Female sex & 2.15 & 0.07 & 0.21 & 3.70 & $0.03^{*}$ & 0.15 & 4.65 \\
\hline Education & -1.25 & 1.10 & -0.08 & -1.14 & $0.26 \mathrm{NS}$ & -3.42 & 0.91 \\
\hline Occupation & -1.38 & 0.56 & -0.15 & -2.44 & $0.22 \mathrm{NS}$ & -2.49 & 0.27 \\
\hline Marital status & 0.42 & 1.01 & 0.02 & 0.42 & $0.68 \mathrm{NS}$ & -1.57 & 2.40 \\
\hline Residence & 0.35 & 1.43 & 0.01 & 0.24 & $0.81 \mathrm{NS}$ & -2.48 & 3.17 \\
\hline Past history of psychiatric disorder & 8.86 & 1.85 & 0.26 & 4.79 & $<0.001^{* *}$ & 5.22 & 12.51 \\
\hline Family history of psychiatric disorders & -0.88 & 1.67 & -0.03 & -0.53 & $0.60 \mathrm{NS}$ & -4.16 & 2.41 \\
\hline Smoking & 0.73 & 1.73 & 0.07 & 0.42 & $0.67 \mathrm{NS}$ & -2.67 & 4.13 \\
\hline Fear of working during COVID-19 & 1.59 & 1.37 & 0.19 & 1.16 & $0.25 \mathrm{NS}$ & 1.15 & 4.28 \\
\hline Fear of infection of COVID-19 & 4.52 & 1.67 & 0.18 & 2.72 & $0.01 *$ & 1.24 & 7.80 \\
\hline Fear of transmission of infection to relatives & 13.07 & 3.76 & 0.31 & 3.48 & $0.001 *$ & 5.66 & 20.48 \\
\hline Had infected with COVID-19 & 0.10 & 1.22 & 0.01 & 0.08 & $0.94 \mathrm{NS}$ & -2.31 & 2.51 \\
\hline Had family member infected with COVID-19 & 0.08 & 1.81 & 0.00 & 0.04 & $0.97 \mathrm{NS}$ & -3.48 & 3.65 \\
\hline Fear of death with COVID-19 & 4.74 & 2.16 & 0.12 & 2.18 & $0.03^{*}$ & 9.02 & 0.46 \\
\hline Fear of family member death of COVID-19 & 4.36 & 1.41 & 0.17 & 3.09 & $0.002^{*}$ & 1.58 & 7.15 \\
\hline Suicidal thoughts & 0.37 & 1.40 & 0.07 & 0.28 & $0.83 \mathrm{NS}$ & -2.44 & 3.26 \\
\hline Working hours & 0.02 & 0.13 & 0.01 & 0.16 & $0.87 \mathrm{NS}$ & -0.24 & 0.28 \\
\hline Sleep hours & -1.10 & 0.38 & -0.16 & -2.86 & $0.005^{*}$ & -1.85 & -0.34 \\
\hline
\end{tabular}

Bold values highlight the significant results

NS non-significant $(P>0.05)$

*Significant $(P<0.05)$; **Highly significant $(P<0.001)$ 


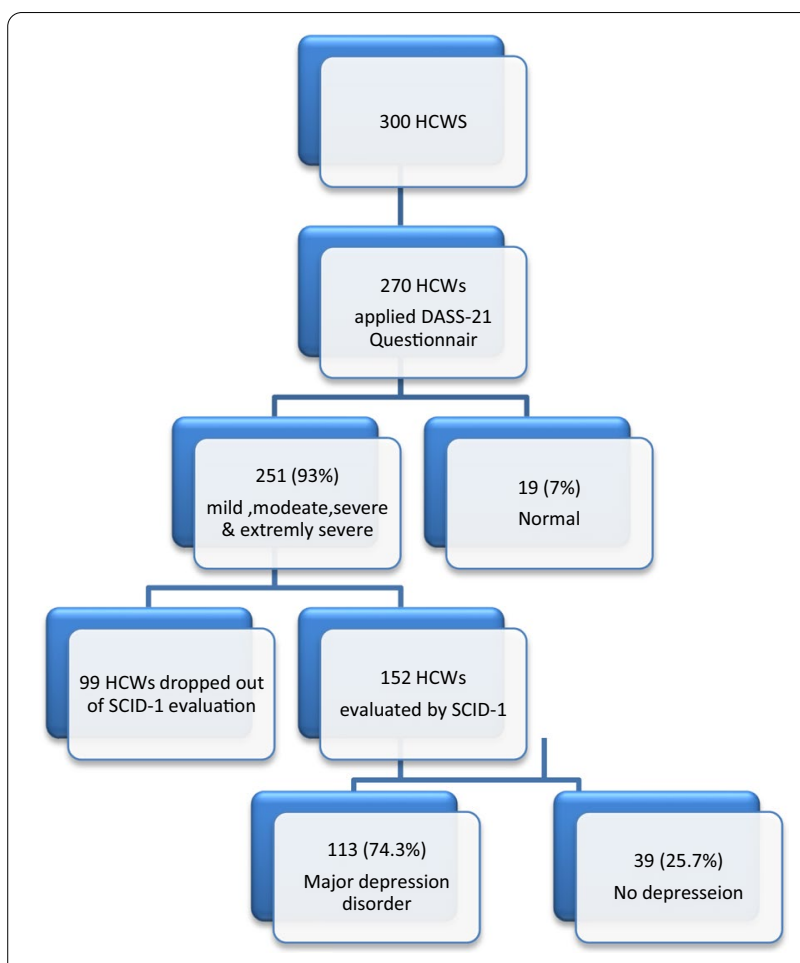

Fig. 2 Diagram for the whole sample

Another Turkish study reported that $64.7 \%$ of physicians had symptoms of depression [20]. Many studies were performed in China: two of them reported that the prevalence of depressive symptoms among health care providers was $50.4 \%$ [21] and $56.0 \%$ [22].

The current results reported higher prevalence of depressive symptoms among HCWs than that of other studies, most probably due to the different conditions in which the HCWs are working in Egypt. HCWs did not deal with such a catastrophic and emerging pandemic before. Moreover, they are facing highly infectious disease with uncertain outcome with deficient infection control supplements and shortage of protective equipment that cause overburden and extraordinary stressors over them [22-25]. In addition, there are other factors that may explain the high prevalence of depression among HCWs generally such as workloads, burnout, insufficient time to take care of their families during the pandemic, social stigma, health anxiety and fear towards COVID-19 infection and reluctance of the society to support them [26-30].

The current study found that young age was associated with higher scores of depression among HCWs. This finding is supported by a recent Jordanian study conducted during the COVID-19 pandemic, which demonstrated that the young age group of HCWs had a significantly high risk to develop depression [16]. A study in Saudi Arabia stated that the age group from 30 to 39 had a slightly high level of depression and anxiety [18]. This is also consistent with recent studies that demonstrate an elevated incidence of psychiatric disorders in younger adults [31,32]. This could be attributed to the less adaptive manner of responding to stressors that may justify this result [33] and the age-related biopsychosocial changes [31]. Moreover, young HCWs had to spend a long time in emergency units in close contact with COVID-19 patients to gain clinical experiences, causing a high level of stress and fear of acquiring the infection.

The results showed that fear of being infected with COVID-19 or transmitting the infection to family members, fear of death, or family members' death with COVID-19, were associated with an increase in the severity of depression among HCWs. In line with these findings, recent studies in China and Nepal reported that medical staffs were fearful about transmitting the virus to their families [21, 23, 28]. A recent study reported that the most concerns regarding the COVID-19-related fears among medical staff in Egypt were the fear of being infected and the fear of transmission of the disease to their families [34]. HCWs may isolate themselves to lower the risk of infecting their family members. Thus the absence of emotional support could attribute the increased psychological distress and affect HCWs mental well-being [35].

The current study found that female HCWs were six times more likely to have depression than male HCWs. Similarly, the results of the Saudi, Jordanian and Egyptian studies indicate that being female increased the risk of depression among health care providers during the COVID-19 pandemic [16, 17, 36, 37]. HCWs with past history of psychiatric illness were seven times more likely to have depression than those without such history according to the current study. In line with this finding, other studies $[23,38]$ reported that health workers who had a history of medication for mental health problems had a higher risk to exhibit anxiety, depression, and insomnia symptoms compared with those who had no psychiatric history.

In addition, HCWs with decrease in sleep hours were two times more likely to have depression, but depression is associated with decrease in sleep hours and the crosssectional design of this study cannot answer what started first?

Finally, HCWs who have fear of COVID-19 infection for themselves or relatives were four times more likely to have depression more than HCWs who did not have this fear. Fear of death for HCWs or their relatives double the risk of depression among HCWs. These findings are supported by previous studies [21, 23, 28]. 
Table 3 Comparison between those who are not depressed versus those who are depressed according to SCID-1 evaluation

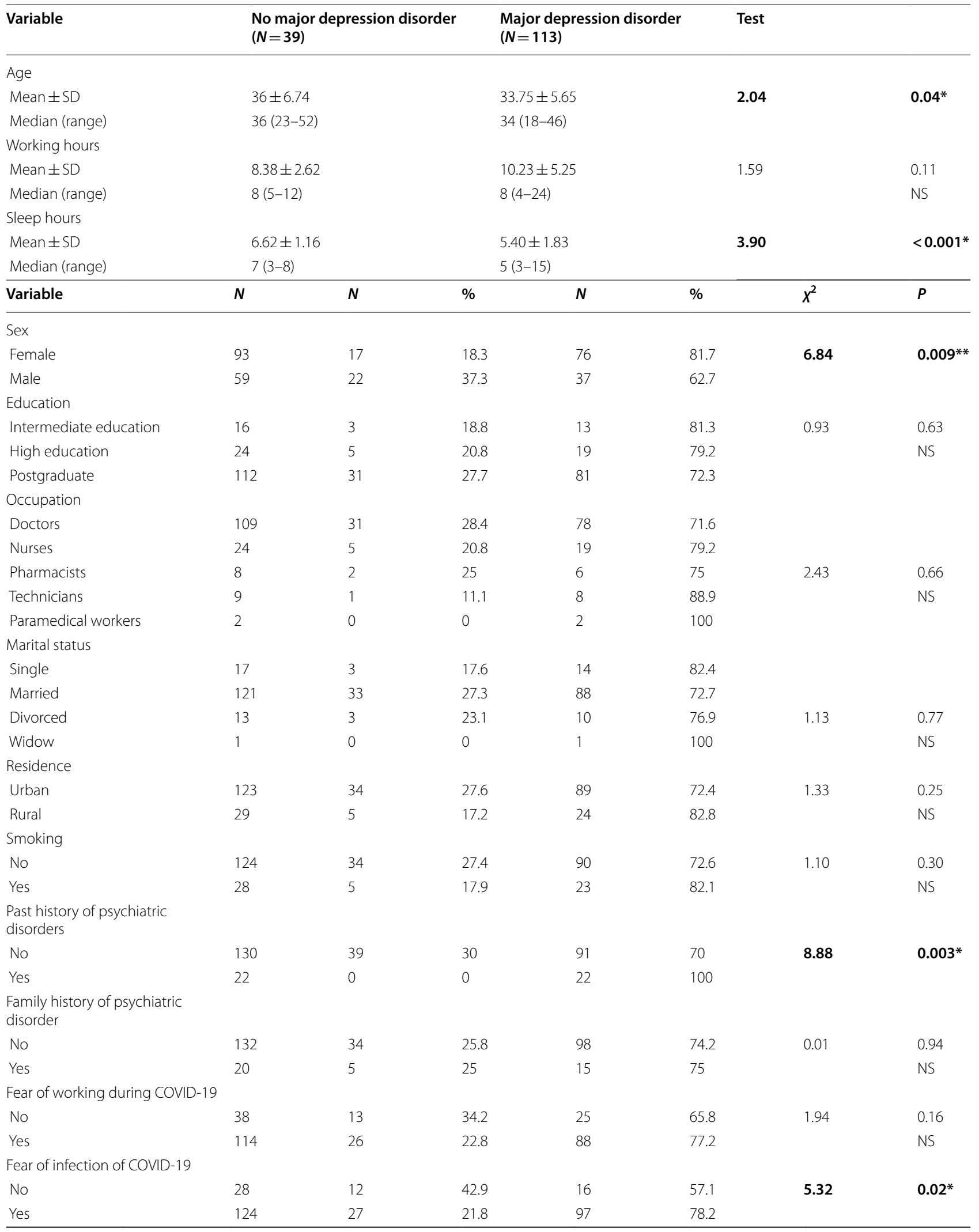


Table 3 (continued)

\begin{tabular}{|c|c|c|c|c|c|c|c|}
\hline Variable & $N$ & $N$ & $\%$ & $N$ & $\%$ & $x^{2}$ & $P$ \\
\hline \multicolumn{8}{|c|}{$\begin{array}{l}\text { Fear of transmission of infection } \\
\text { to relatives }\end{array}$} \\
\hline No & 5 & 4 & 80 & 1 & 20 & 8.00 & $0.005^{*}$ \\
\hline Yes & 147 & 35 & 23.8 & 112 & 76.2 & & \\
\hline \multicolumn{8}{|c|}{ Had infected with COVID-19 } \\
\hline No & 129 & 36 & 27.9 & 93 & 72.1 & 2.26 & 0.13 \\
\hline Yes & 23 & 3 & 13 & 20 & 87 & & NS \\
\hline \multicolumn{8}{|c|}{$\begin{array}{l}\text { Had family member infected with } \\
\text { COVID-19 }\end{array}$} \\
\hline No & 12 & 1 & 8.3 & 11 & 91.7 & 2.05 & 0.15 \\
\hline Yes & 140 & 38 & 27.1 & 102 & 72.9 & & NS \\
\hline \multicolumn{8}{|c|}{ Fear of death with COVID-19 } \\
\hline No & 108 & 33 & 30.6 & 75 & 69.46 & 3.94 & $0.03^{*}$ \\
\hline Yes & 44 & 6 & 13.6 & 38 & 86.4 & & \\
\hline \multicolumn{8}{|c|}{$\begin{array}{l}\text { Fear of death of family member } \\
\text { with COVID-19 }\end{array}$} \\
\hline No & 47 & 17 & 36.2 & 30 & 63.8 & 4.69 & $0.04^{*}$ \\
\hline Yes & 105 & 22 & 21 & 83 & 79 & & \\
\hline \multicolumn{8}{|c|}{ Suicidal thoughts } \\
\hline No & 149 & 47 & 31.5 & 102 & 68.5 & 1.37 & 0.24 \\
\hline Yes & 3 & 0 & 0 & 3 & 100 & & NS \\
\hline
\end{tabular}

Bold values highlight the significant results

$S D$ standard deviation, Test independent t test, $\chi^{2}$ chi-square test, NS non-significant $(P>0.05)$

*Significant $(P<0.05)$; **Highly significant $(P<0.001)$

Table 4 Binary logistic regression analysis for predictors of major depression disorder according to SCID-1 among the studied group

\begin{tabular}{|c|c|c|c|c|c|c|c|}
\hline & $B$ & S.E & Wald & $P$ & OR & $95 \% \mathrm{C}$ & \\
\hline Young age & 0.24 & 0.08 & 8.04 & $0.005^{*}$ & 1.27 & 1.08 & 4.49 \\
\hline Female sex & 2.36 & 0.90 & 6.88 & $0.009^{*}$ & 6.25 & 3.13 & 18.36 \\
\hline Education & 1.16 & 1.59 & 0.54 & $0.47 \mathrm{NS}$ & 3.19 & 0.14 & 71.80 \\
\hline Occupation & -0.53 & 2.87 & 0.03 & $0.85 \mathrm{NS}$ & 0.59 & 0.002 & 164.62 \\
\hline Marital status & 0.32 & 2.23 & 0.02 & $0.89 \mathrm{NS}$ & 1.38 & 0.02 & 108.49 \\
\hline Past history of a psychiatric disorder & 6.06 & 2.43 & 6.20 & $0.01 *$ & 7.63 & 3.64 & 9.62 \\
\hline Residency & 2.06 & 2.66 & 2.30 & $0.91 \mathrm{NS}$ & 1.19 & 0.04 & 32.33 \\
\hline Family history of psychiatric disorder & -0.63 & 1.00 & 0.39 & $0.53 \mathrm{NS}$ & 0.53 & 0.08 & 3.80 \\
\hline Smoking & 1.53 & 1.06 & 2.09 & $0.15 \mathrm{NS}$ & 4.63 & 0.58 & 36.97 \\
\hline Fear of working with during COVID-19 & 0.50 & 0.80 & 0.39 & $0.53 \mathrm{NS}$ & 1.65 & 0.35 & 7.85 \\
\hline Fear of infection with COVID-19 & 2.25 & 1.10 & 4.17 & $0.04^{*}$ & 3.95 & 1.09 & 5.88 \\
\hline Fear of transmission of COVID-19 to a family member & 2.83 & 1.32 & 4.65 & 0.02 & 4.11 & 1.23 & 6.71 \\
\hline Had infected of COVID-19 & 1.38 & 1.11 & 1.56 & $0.21 \mathrm{NS}$ & 3.99 & 0.45 & 35.19 \\
\hline Had a family member infected with COVID-19 & 0.44 & 0.81 & 0.30 & $0.59 \mathrm{NS}$ & 1.55 & 0.32 & 7.58 \\
\hline Fear of death with COVID-19 & 1.85 & 1.02 & 3.26 & $0.03^{*}$ & 2.16 & 1.04 & 5.17 \\
\hline Fear of family member death of COVID-19 & 1.79 & 1.01 & 3.09 & 0.04 & 2.01 & 1.02 & 4.93 \\
\hline Suicidal thoughts & 1.13 & 1.20 & 2.50 & $0.70 \mathrm{NS}$ & 2.16 & 0.73 & 8.12 \\
\hline Working hours & 0.12 & 0.07 & 2.82 & $0.09 \mathrm{NS}$ & 1.13 & 0.98 & 1.31 \\
\hline Sleep hours & 0.69 & 0.29 & 5.62 & $0.018^{*}$ & 2.00 & 1.13 & 3.55 \\
\hline
\end{tabular}

Bold values highlight the significant results

NS non-significant $(P>0.05)$

*Significant $(P<0.05)$; ${ }^{*}$ Highly significant $(P<0.001)$ 
According to these findings, the mental health status of HCWs should be closely monitored by the Ministry of Health to facilitate the appropriate psychological care. They should be provided with appropriate safety measures, their workload should be managed and they should be compensated by the appropriate financial support, to reduce the mental health burden during such pandemic. This study had some limitations which include: first, cross-sectional design does not investigate causality; second, only $56 \%$ of the sample size was interviewed using SCID-I, which might limit the strength of using a structural interview instead of selfrating scales. However, this study has some strengths: first, it used structural interview based on SCID-I in addition to self-rating scales; second, it includes many health professions not only physicians.

\section{Conclusions}

Three out of four HCWs who were caring for COVID19 patients had major depression and two-thirds had severe symptoms. Young age, female gender, decreased sleep hours, past history of psychiatric illness, fear of infection or death due to COVID-19 were not only predictors of depression, but also of its severity. Screening for depression among HCWs, particularly for young females, and early treatment are recommended.

\section{Abbreviations}

SCID: Structure Clinical Interview of DSM; HCWs: Healthcare workers; MDD: Major depression disorder; DASS: Depression Anxiety Stress Scale; WHO: World Health Organization; PPE: Personal protective equipment.

\section{Acknowledgements}

We would like to thank all the participants for their valued time.

\section{Authors' contributions}

HE: writing and editing. MS: interpretation and analysis of the data and writing. MB: work design. UY: collecting data and revision. DE: statistical analysis and writing. SA: data collection and writing. All authors read and approved the final manuscript.

\section{Funding}

This research did not receive any specific grant from funding agencies in the public, commercial, or not-for-profit sectors.

\section{Availability of data and materials}

Available upon request.

\section{Declarations}

Ethics approval and consent to participate

The study was approved by Zagazig University institutional review board (IRB No. 6216). All study procedures were conducted within the ethical guidelines as outlined in the Declaration of Helsinki and its later amendments. All participants signed a written consent.

\section{Consent for publication}

Not available.

\section{Competing interests}

There are no competing interests.

\section{Author details}

${ }^{1}$ Department of Psychiatry, Faculty of Medicine, Zagazig University, PO Box 44519, Zagazig, Egypt. ${ }^{2}$ Department of Community, Environmental and Occupational Medicine, Faculty of Medicine, Zagazig University, Zagazig, Egypt.

Received: 11 June 2021 Accepted: 8 October 2021

Published online: 18 October 2021

\section{References}

1. Murdoch DR, Howie SR. The global burden of lower respiratory infections: making progress, but we need to do better. Lancet Infect Dis. 2018;18(11):1162-3.

2. Huang C, et al. Clinical features of patients infected with 2019 novel coronavirus in Wuhan, China. Lancet. 2020;395(10223):497-506.

3. Liu X, et al. Depression after exposure to stressful events: lessons learned from the severe acute respiratory syndrome epidemic. Compr Psychiatry. 2012;53(1):15-23.

4. Lung F-W, et al. Mental symptoms in different health professionals during the SARS attack: a follow-up study. Psychiatr Q. 2009;80(2):107.

5. Wu P, et al. The psychological impact of the SARS epidemic on hospital employees in China: exposure, risk perception, and altruistic acceptance of risk. Can J Psychiatry. 2009:54(5):302-11.

6. Fava GA, et al. Clinical characterization of allostatic overload. Psychoneuroendocrinology. 2019;108:94-101.

7. Bao Y, et al. 2019-nCoV epidemic: address mental health care to empower society. Lancet. 2020;395(10224):e37-8.

8. Chen Q, et al. Mental health care for medical staff in China during the COVID-19 outbreak. Lancet Psychiatry. 2020;7(4):e15-6.

9. Maunder RG, et al. Factors associated with the psychological impact of severe acute respiratory syndrome on nurses and other hospital workers in Toronto. Psychosom Med. 2004;66(6):938-42.

10 Pappa S, et al. Prevalence of depression, anxiety, and insomnia among healthcare workers during the COVID-19 pandemic: a systematic review and meta-analysis. Brain Behav Immunity. 2020;88:901-7.

11 Dean AG, et al. Epi Info: a word-processing, database, and statistics program for public health on IBM-compatible microcomputers programming. Atlanta: Centers for Disease Control and Prevention; 1995.

12. Lovibond PF, Lovibond SH. The structure of negative emotional states: comparison of the Depression Anxiety Stress Scales (DASS) with the Beck Depression and Anxiety Inventories. Behav Res Ther. 1995;33(3):335-43.

13. First MB, et al. SCID-5-CV: structured clinical interview for DSM-5 disorders, clinician version. Arlington: American Psychiatric Association Publishing; 2016.

14. Chua SE, et al. Psychological effects of the SARS outbreak in Hong Kong on high-risk health care workers. Can J Psychiatry. 2004;49(6):391-3.

15. Khalaf OO, Khalil MA, Abdelmaksoud R. Coping with depression and anxiety in Egyptian physicians during COVID-19 pandemic. MECP. 2020;27(1):1-7.

16 Naser AY, et al. Mental health status of the general population, healthcare professionals, and university students during 2019 coronavirus disease outbreak in Jordan: a cross-sectional study. Brain Behav. 2020;10(8):e01730.

17. Ahmed GK, et al. Comparison of knowledge, attitude, socioeconomic burden, and mental health disorders of COVID-19 pandemic between general population and health care workers in Egypt. Egypt J Neurol Psychiatr Neurosurg. 2021;57(1):1-11.

18. AlAteeq $D A$, et al. Mental health among healthcare providers during coronavirus disease (COVID-19) outbreak in Saudi Arabia. J Infect Public Health. 2020;13(10):1432-7.

19. Aly HM, et al. Stress, anxiety and depression among healthcare workers facing COVID-19 pandemic in Egypt: a cross-sectional online-based study. BMJ Open. 2021;11(4):e045281.

20. Elbay RY, et al. Depression, anxiety, stress levels of physicians and associated factors in Covid-19 pandemics. Psychiatry Res. 2020;290:113130. 
21. Lai J, et al. Factors associated with mental health outcomes among health care workers exposed to coronavirus disease 2019. JAMA Netw Open. 2020;3(3):e203976-e203976.

22. Xing L-q, et al. Anxiety and depression in frontline health care workers during the outbreak of Covid-19. Int J Soc Psychiatry. 2020. https://doi. org/10.1177/0020764020968119.

23. Khanal $P$, et al. Mental health impacts among health workers during COVID-19 in a low resource setting: a cross-sectional survey from Nepal. Glob Health. 2020;16(1):1-12.

24. Li Q, et al. Early transmission dynamics in Wuhan, China, of novel coronavirus-infected pneumonia. N Engl J Med. 2020;382:1199-207.

25. Rothe C, et al. Transmission of 2019-nCoV infection from an asymptomatic contact in Germany. N Engl J Med. 2020;382(10):970-1.

26. Abdelghani $M$, et al. Addressing the relationship between perceived fear of COVID-19 virus infection and emergence of burnout symptoms in a sample of Egyptian physicians during COVID-19 pandemic: a crosssectional study. MECP. 2020;27(1):70.

27. Chan-Yeung M. Severe acute respiratory syndrome (SARS) and healthcare workers. Int J Occup Med Environ Health. 2004;10(4):421-7.

28 Chen W, Huang Y. To protect health care workers better, to save more lives with COVID-19. Anesth Analg. 2020. https://doi.org/10.1213/ANE. 0000000000004834

29. Abdelghani M, Mahdy R, El-Gohari H. Health anxiety to COVID-19 virus infection and its relationship to quality of life in a sample of health care workers in Egypt: a cross-sectional study. Arch Psychiatry Psychother. 2021;23(1):19-28.

30 Urooj U, et al. Expectations, fears and perceptions of doctors during Covid-19 pandemic. Pak J Med Sci. 2020;36(COVID19-S4):S37.
31. Flint AJ, et al. Effect of age on the frequency of anxiety disorders in major depression with psychotic features. Am J Geriatr Psychiatry. 2010;18(5):404-12.

32. Wells JE, et al. Prevalence, interference with life and severity of 12 month DSM-IV disorders in Te Rau Hinengaro: the New Zealand mental health survey. Aust N Z J Psychiatry. 2006;40(10):845-54.

33. Schilling OK, Diehl M. Psychological vulnerability to daily stressors in old age. Z Gerontol Geriatr. 2015;48(6):517-23.

34. Wahed WYA, et al. Assessment of knowledge, attitudes, and perception of health care workers regarding COVID-19, a cross-sectional study from Egypt. J Community Health. 2020;45(6):1242-51.

35. QasemSurrati AM, AsadMansuri FM, AyadhAlihabi AA. Psychological impact of the COVID-19 pandemic on health care workers. J Taibah Univ Med Sci. 2020;15(6):536-43.

36 Alkhamees AA, et al. The psychological impact of COVID-19 pandemic on the general population of Saudi Arabia. Compr Psychiatry. 2020;102:152192.

37. Youssef $\mathrm{N}$, et al. Mental health status of health-care professionals working in quarantine and non-quarantine Egyptian hospitals during the COVID19 pandemic. East Mediterr Health J. 2020:26:1155-64.

38. Zhu Z, et al. COVID-19 in Wuhan: sociodemographic characteristics and hospital support measures associated with the immediate psychological impact on healthcare workers. EClinicalMedicine. 2020;24:100443.

\section{Publisher's Note}

Springer Nature remains neutral with regard to jurisdictional claims in published maps and institutional affiliations.

\section{Submit your manuscript to a SpringerOpen ${ }^{\circ}$ journal and benefit from:}

- Convenient online submission

- Rigorous peer review

- Open access: articles freely available online

- High visibility within the field

- Retaining the copyright to your article

Submit your next manuscript at springeropen.com 